\title{
Response to Dr. Netanel Berko's letter entitled "Partial anomalous pulmonary venous return: more common from the left or right lung?"
}

\author{
Keishi Okamoto · Tetsuaki Wakebe • \\ Kazunobu Saiki
}

Received: 6 July 2009/ Accepted: 10 July 2009/Published online: 15 August 2009

(C) Japanese Association of Anatomists 2009

\section{Dear Sir,}

In his letter, Dr. Netanel Berko points out a difference between the previous studies cited in our report (Okamoto et al. 2008) and the recent study based on computed tomography (Haramati et al. 2003) regarding the occurrence of partial anomalous pulmonary venous return. Based on the results of two early studies (Brody 1942; Snellen et al. 1968), the most frequent type of partial anomalous pulmonary venous drainage is that from the right upper lobe to the superior vena cava. In contrast, the Haramati study demonstrated conflicting computed tomographic (CT) findings in which an anomalous left upper lobe vein draining into a persistent left vertical vein was the most frequent type. We agree with Dr. Berko's explanation that this difference in prevalence may be attributed to the patient population studied as well as the underdetection of partial anomalous pulmonary venous drainage from the right upper lobe by CT (Haramati et al. 2003). In particular, we consider the latter explanation as being more likely based on the observation that when we select an adult population from the list of cases studied by Brody in 1942, omitting the younger patients, the most frequent type of partial anomalous pulmonary venous drainage remains the same. We have encountered three cases of partial anomalous pulmonary venous return among approximately 800 adult Japanese cadavers. In two of these cases, the anomalous pulmonary vein drained into the superior vena cava from the right upper lobe, and in one case, it drained into the left brachiocephalic vein from the left upper lobe (Okamoto et al. 2004, 2008). Therefore, the actual frequency of each type of partial anomalous pulmonary venous return, especially using $\mathrm{CT}$, remains to be clarified. In addition, we would like to emphasize that the main purpose of our report was to demonstrate the role of the bronchial vein as a boundary vein between pulmonary and systemic circulations. Our reference to the frequent type of partial anomalous pulmonary venous return was intended to suggest that the occurrence of such an anomaly has a morphological background.

\section{References}

Brody H (1942) Drainage of the pulmonary veins into the right side of the heart. Arch Pathol 33:221-240

Haramati LB, Moche IE, Rivera VT et al (2003) Computed tomography of partial anomalous pulmonary venous connection in adults. J Comput Assist Tomogr 27:743-749

Okamoto K, Kodama K, Kawai K, Wakebe T, Saiki K, Nagashima S (2004) An anatomical study of the partial anomalous pulmonary venous return with special references to the bronchial vein. Anat Sci Int 79:82-86

Okamoto K, Wakebe T, Saiki K (2008) Anatomical study of the partial anomalous pulmonary venous return with special references to the bronchial vein: supplementary report. Anat Sci Int 83:169-172

Snellen HA, van Ingen HC, Hoefsmit ECHM (1968) Patterns of anomalous pulmonary venous drainage. Circulation 38:45-63

\author{
K. Okamoto $(\bowtie) \cdot$ T. Wakebe $\cdot$ K. Saiki \\ Department of Macroscopic Morphology, Unit of Basic Medical \\ Sciences, Graduate School of Biomedical Science, \\ Nagasaki University, 1-12-4 Sakamoto, \\ Nagasaki 852-8523, Japan \\ e-mail: okamon@nagasaki-u.ac.jp
}

